\title{
MAXIMAL-CLIQUE PARTITIONS OF INTERVAL GRAPHS
}

\author{
MA SHAOHAN and W. D. WALLIS
}

(Received 8 October 1986)

Communicated by Louis Caccetta

\begin{abstract}
It is shown that if an interval graph possesses a maximal-clique partition then its clique covering and clique partition numbers are equal, and equal to the maximal-clique partition number. Moreover an interval graph has such a partition if and only if all its maximal cliques are edge-disjoint .
\end{abstract}

1980 Mathematics subject classification (Amer. Math. Soc.): 05 C 35.

\section{Interval graphs and clique-matrices}

Throughout this paper graphs are finite, undirected, loopless and without multiple edges. A clique is a complete subgraph, and a maximal clique is a clique which is not a proper subgraph of any other clique.

A graph $G$ is called an interval graph if its vertices can be put into one-to-one correspondence with a set of intervals $\mathcal{F}$ of the real line, such that two vertices are connected by an edge of $G$ if and only if the corresponding intervals have nonempty intersection. Clearly any induced subgraph of an interval graph is an interval graph.

The earliest characterization of interval graphs was obtained by Lekkerkerker and Boland [3], as follows.

The second author's research was supported by NSF grant DMS 86-01828.

(C) 1988 Australian Mathematical Society 0263-6115/88 \$A2.00+0.00 
LEMMA 1. An undirected graph $G$ is an interval graph if and only if the following two conditions are satisfied:

I1. $G$ is a triangulated graph (that is, every 4-cycle in $G$ contains a chord);

I2. Any triple of vertices of $G$ can be ordered in such a way that every path from the first vertex to the third vertex passes through a neighbor of the second vertex.

Three vertices which fail to satisfy condition $\mathrm{I} 2$ are called an asteroidal triple. For example, the graph $G_{1}$ in Figure 1 has an asteriodal triple $\{a, c, e\}$, whence $G_{1}$ is not interval graph. But the graph $G_{2}$ in Figure 1 is an interval graph.
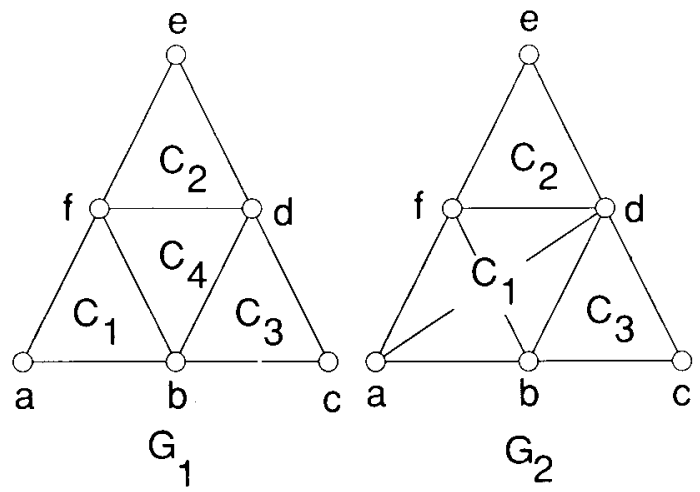

FIGURE 1

Thus $G$ is an interval graph if and only if $G$ is a triangulated graph which contains no asteroidal triple. So it follows from [3] that an interval graph $G=(V, E)$ has at most $|V|$ maximal cliques with equality if and only if $G$ has no edges, and those maximal cliques can be found in $O(|V|+|E|)$ time.

$$
\begin{aligned}
& \begin{array}{llllll}
a & b & c & d & e & f
\end{array}
\end{aligned}
$$

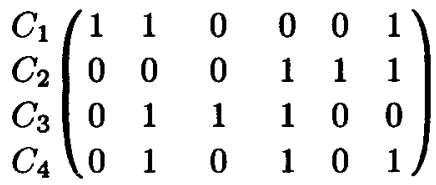

$$
\begin{aligned}
& M_{1} \\
& \begin{array}{lllllllllllll}
a & b & c & d & e & f & a & b & c & d & e & f
\end{array} \\
& \begin{array}{l}
C_{1} \\
C_{2} \\
C_{3}
\end{array}\left(\begin{array}{llllll}
1 & 1 & 0 & 1 & 0 & 1 \\
0 & 0 & 0 & 1 & 1 & 1 \\
0 & 1 & 1 & 1 & 0 & 0
\end{array}\right) \rightarrow \begin{array}{l}
C_{3} \\
C_{1} \\
C_{2}
\end{array}\left(\begin{array}{llllll}
0 & 1 & 1 & 1 & 0 & 0 \\
1 & 1 & 0 & 1 & 0 & 1 \\
0 & 0 & 0 & 1 & 1 & 1
\end{array}\right) \\
& M_{2} \\
& M_{2}^{\prime}
\end{aligned}
$$

FIGURE 2 
Suppose the graph $G$ has maximal cliques $C_{1}, C_{2}, \ldots$ The clique matrix $M=\left(m_{i j}\right)$ of $G$ is a $(0,1)$-matrix in which $m_{i j}=1$ and only if $C_{i}$ contains the $j$ th vertex, $v_{j}$ say, of $G$. A $(0,1)$-matrix $M$ is said to have the consecutive ones property for columns if its rows can be permuted in such a way that the $1 \mathrm{~s}$ in each column appear consecutively. For example, suppose $M_{1}$ and $M_{2}$ are the clique-matrices of the graphs $G_{1}$ and $G_{2}$ of Figure 1; they are shown in Figure 2. The permutation (123) of the rows of $M_{2}$ produces a matrix $M_{2}^{\prime}$ of the required form, so $M_{2}$ has the consecutive ones property; but $M_{1}$ does not.

LEMMA 2 [2]. An undirected graph $G$ is an interval graph if and only if its clique matrix $M$ has the consecutive ones property for columns. Equivalently, the maximal cliques of $G$ can be linearly ordered such that, for every vertex $v$ of $G$, the maximal cliques containing $v$ occur consecutively.

REMARK. Lemma 2 clearly suggests a recognition algorithm for interval graphs $G=(V, E)$ by a two-step process. First one checks whether $G$ is triangulated and if so, enumerates its maximal cliques. This can be executed in $O(|V|+|E|)$ time and will produce at most $|V|$ maximal cliques. Second, one tests whether or not the clique-matrix of $G$ has the consecutive ones property for columns, the data structure needed to solve the consecutive ones problem most efficiently is the $P Q$-tree; $P Q$-trees were invented by Booth and Leuker [1] expressly for this purpose.

\section{Maximal clique partitions of interval graphs}

A clique covering of a graph $G$ is a family $C$ of cliques of $G$ such that every edge of $G$ lies in some member of $C$. If the clique covering $C$ has cardinality $|C|$ and $\left|C X^{\prime}\right| \geq|C|$ for all clique coverings $C^{\prime}$ of $G$, then $C$ is called a minimal clique covering and the clique covering number of $G, c c(G)$, is defined to equal $|C|$. A clique covering whose members are disjoint is called a clique partition; the clique partition number $c p(G)$ is defined to be the smallest possible cardinality of clique partitions of $G$. If $G$ has no edges, we define $c p(G)=c c(G)=0$. A maximalclique partition is a clique partition into maximal cliques; many graphs have no such partition-for example, if $n \geq 4$ the graph derived from $K_{n}$ by deleting one edge has no maximal clique partition. But, if a graph $G$ has a maximalclique partition, its maximal-clique partition number $m c p(G)$ is defined to be the minimum of the cardinalities of maximal-clique partitions. For convenience we write $\operatorname{mcp}(G)=0$ if $G$ has no edges. Clearly whenever $m c p(G)$ is defined we have $c c(G) \leq c p(G) \leq m c p(G)$, with equality, for example, when $G$ is triangle-free. 
Suppose $H$ is a subgraph of $G$ with the property that, for any clique $K$ of $G$, either every edge of $K$ or no edge of $K$ lies in $H$. Then we say that " $H$ separates the cliques of $G^{\prime \prime}$. We say that $G$ is clique-separable if there exists some proper, non-empty subgraph $H$ of $G$ which separates the cliques of $G$; otherwise $G$ is clique-inseparable. If the subgraph $B$ of $G$ separates the cliques of $G$ but no proper non-empty subgraph of $B$ does so, we call $B$ a clique-block of $G$. Note that such a graph $B$ is itself clique-inseparable.

LEMMA 3 [5, LEMMA 2.7]. The clique-blocks of a graph $G$ partition the edge-set of $G$.

LEMMA 4 [6, LEMMA 2.1]. G has a maximal-clique partition if and only if each of its clique-blocks has a maximal-clique partition. In that case

$$
m c p(G)=\sum m c p(B)
$$

where the sum is taken over all clique-blocks $B$ of $G$.

For further discussion of maximal-clique partitions, clique-inseparability and clique-blocks, the reader is referred to [6].

LEMMA 5. If $G$ is an interval graph, then any clique-block $B$ of $G$ is an induced subgraph of $G$.

ProOF. If $B$ is not an induced subgraph of $G$, then there exist two vertices $u, v$ in $B$ such that $(u, v)$ is an edge of $G$ but not of $B$. Because $B$ is a connected subgraph of $G$, there exists a path between $u$ and $v$ in $B$. Moreover, since $G$ is an interval graph (and so it contains no cordless 4-cycle), there exists a shortest path $P$ between $u$ and $v$ in $B$, whose length is exactly 2 . Thus the path $P$ and edge $(u, v)$ form a triangle. But $P \in B,(u, v) \notin B$. This contradicts the cliqueblock property of $B$ so the edge $(u, v)$ must belong to $B$ and $B$ is a (vertex) induced subgraph of $G$.

COROLLARY. If $G$ is an interval graph and $B$ is any clique-block of $G$, then $B$ is an interval graph.

PrOOF. It suffices to observe that any induced subgraph of an interval graph is again an interval graph.

But suppose $G$ is an interval graph and $B$ is any induced subgraph. The incidence matrix of $G$ has the consecutive ones property, by Lemma 2. Suppose the rows have been ordered in such a way that the ones in each column appear consecutively; assume the columns to have been reordered in the same way. Then we still have the incidence matrix of $G$, after vertices have been reordered. The 
passage to an induced subgraph involves deletion of certain rows and columns, and this does not disturb the consecutive ones property. So $B$ is also an interval graph, from Lemma 2.

LEMMA 6. If $G$ is an interval graph, then $G$ has a maximal-clique partition if and only if its clique-blocks are all cliques.

PrOOF. If $G$ is an interval graph and has a maximal-clique partition, then its clique-blocks are also interval graphs. From Lemma 4 each clique-block also has a maximal-clique partition. Suppose some clique-block $B$ is not itself a clique. Then $B$ has a maximal-clique partition, say $C_{1} \cup C_{2} \cup \cdots \cup C_{k}$. If $C_{1}$ is disjoint from $C_{2} \cup C_{3} \cup \cdots \cup C_{k}$ then $C_{2} \cup C_{3} \cup \cdots \cup C_{k}$ separates the cliques of $B$; since $B$ is itself clique-inseparable, this is impossible. So $C_{1}$ has a vertex in common with some other clique. Say $C_{1}$ and $C_{2}$ meet in $y_{1}$. (They cannot meet in more than one point, since $C_{1} \cup C_{2}$ can contain no edge.) Similarly, we can find vertices $y_{2}, y_{3}, \ldots, y_{h}$ such that $y_{2}=C_{2} \cap C_{3}, y_{3}=C_{3} \cap C_{4}, \ldots$, and $y_{h}=C_{h} \cap C_{1}$. We do not care whether $h=k$ or $h<k$. However it is important to observe that $h \geq 3$ : the case $h=2$ is clearly impossible and if $h=1$ is the only possibility, meaning that $B$ consists of two cliques with a common vertex, we again see that $B$ is clique-separable. So the clique-matrix of $B$ contains a submatrix

\begin{tabular}{|c|c|c|c|}
\hline & $y_{1}$ & $y_{2}$ & \\
\hline$C_{1}$ & 1 & 0 & \\
\hline$C_{2}$ & 1 & 1 & \\
\hline$C_{3}$ & 0 & 1 & \\
\hline$\dot{C}_{h}$ & & $\ddot{0}$ & \\
\hline
\end{tabular}

which means the matrix does not have the consecutive ones property. This contradicts Lemma 2.

Conversely, if each clique-block is a clique, then obviously every clique-block is a maximal-clique partition. So by Lemma $4 G$ has a maximal-clique partition.

We are now ready to prove the main results.

THEOREM 1. Let $G$ be an interval graph which possesses a maximal-clique partition. Then

$$
c c(G)=c p(G)=\operatorname{mcp}(G)
$$

and every maximal clique lies in the maximal-clique partition.

Proof. From Lemma 6, the clique-blocks of $G$ are all cliques. From the definition of clique-blocks they are all maximal cliques, and from Lemma 3 they partition the edges of $G$. So they form a clique covering, a clique partition 
and a maximal-clique partition. To prove equation (1), it suffices to observe that this clique covering is minimum. But every clique lies entirely within some clique-block, so the number of cliques required to cover $G$ must at least equal the number of clique-blocks.

Since every clique lies in some clique-block, any maximal clique $C$ must lie entirely within some clique-block $B$. As $B$ is a clique, $C=B$, so $C$ lies in the maximal-clique partition.

THEOREM 2. Let $G$ be an interval graph. $G$ has a maximal-clique partition if and only if it has no two maximal cliques whose intersection contains two or more vertices.

PrOOF. The "if" part is obvious.

Suppose maximal cliques $C_{i}$ and $C_{j}$ of a graph $G$ have common edge $e$. Then the clique-block which contains $e$ is not a clique, so by Lemma $6, G$ is not an interval graph.

To see that this theorem is not true for general graphs, observe the graph $C_{1}$ of Figure 1: the maximal cliques $C_{3}$ and $C_{4}$ have a common edge, yet $G_{1}$ has a maximal-clique partition $C_{1} \cup C_{2} \cup C_{3}$.

\section{References}

[1] K. S. Booth and G. S Leuker, 'Testing for the consecutive ones property, interval graphs, and graph planarity using $P Q$-tree algorithms,' J. Comput. System Sci. 13 (1976), 335379.

[2] D. R. Fulkerson and O. A. Gross, 'Incidence matrices and interval graphs,' Pacific J. Math. 15 (1965), 835-855.

[3] C. G. Lekkerkerker and J. Ch. Boland, 'Representation of a finite graph by a set of intervals on the real line,' Fund. Math. 51 (1962), 45-64.

[4] Ma Shaohan and W. D. Wallis, 'Clique partitions of triangulated graphs,' Congress. Numer., to appear.

[5] N. J. Pullman, 'Clique coverings of graphs IV: algorithms,' SIAM J. Comput. 13 (1984), $57-75$.

[6] N. J. Pullman, H. Shank and W. D. Wallis, 'Clique coverings of graphs V: maximal-clique partitions,' Bull. Austral. Math. Soc. 25 (1982), 337-356.

Department of Computer Science

Shandong University

Jinan

People's Republic of China
Department of Mathematics

Southern Illinois University

Carbondale, Illinois 62901

U.S.A. 